\title{
PERFIL, RETOS Y DESAFÍOS DEL ESTUDIANTE UNIVERSITARIO EN EL SIGLO XXI
}

\section{PROFILE, CHALLENGES AND CHALLENGES OF THE UNIVERSITY STUDENT IN THE XXI CENTURY}

\section{Claudia Marcela Durán Chinchilla ${ }^{1}$}

\section{Doris Cecilia Páez Quintero ${ }^{2}$}

\section{Christian Nolasco Serna ${ }^{3}$}

\section{RESUMEN}

La globalización, los medios de información y comunicación, la web 2.0 y la revolución industrial, ha generado cambios metodológicos que intentan favorecer el autoaprendizaje; es así que la educación no se centra en la enseñanza sino en el aprendizaje; el educador, deja de ser transmisor y pasa a ser orientador o dinamizador; el alumno por su parte, se $1 \quad$ Doctora en Educación, Magister en Pedagogía, Especialista en Práctica Pedagógica, Licenciada en lingüística. Docente Universidad Francisco de Paula Santander Ocaña .Investigadora asociada y directora del grupo de investigación GIFEAH cmduranc@ufpso.edu.co. https://orcid. org/0000-0001-9291-7841.

2 MSc, en Dirección Universitaria, docente Universidad Francisco de Paula Santander Ocaña, investigadora grupo de investigación GIFEAH; dcpaezq@ufpso.edu.co, http://orcid.org/000-0003-1418-8860

$3 \quad P h D$ en ciencias matemáticas, docente Universidad Francisco de Paula Santander Ocaña; investigador grupo GIFEAH; cnolascos@ufpso.edu.co, https://orcid. org/0000-0001-6923-3388 convierte en protagonista y autogestionador del conocimiento. A la luz de ello, es importante que el estudiante, siendo protagonista de su aprendizaje, reflexione en torno al mismo proceso en el que está sumergido. Este artículo, intenta perfilar al estudiante universitario del siglo $X X I$, en tal sentido, en primer lugar, se realiza una revisión conceptual acerca de las distintas definiciones y características que se le atribuye al estudiante, sus retos y desafíos.

\section{PALABRAS CLAVE}

Estudiante, perfil, reto y desafío

\section{ABSTRAC}

Globalization, means of information and communication, the web 2.0 and the industrial revolution have generated methodological 
changes that facilitate self- learning. Thus, education is not focused on teaching but on learning. The educator ceases to be a transmitter and becomes a guide or facilitator. On the other hand, students become protagonists and selfmanagers of knowledge. Considering this, it is important that the pupil, as protagonist of his or her learning, reflects on process in which he or she is immersed. The main goal of this article is to evaluate 21 st century college student profiles. A conceptual review is made about the different definitions and characteristics that are attributed to the student as well as their challenges.

KEYWORDS Student, profile, challenge and challenge

\section{RESUMO}

A globalização, os meios de informação e comunicação, a web 2.0 e a revolução industrial têm gerado mudanças metodológicas que procuram favorecer a autoaprendizagem; Assim, a educação não está voltada para o ensino, mas para a aprendizagem; o educador deixa de ser transmissor e passa a ser guia ou facilitador; o aluno, por sua vez, passa a ser o protagonista e autogestionário do conhecimento. Por isso é importante que o aluno, sendo o protagonista de sua aprendizagem, reflita sobre o mesmo processo em que está imerso. Este artigo procura delinear o estudante universitário do século XXI, assim, em primeiro lugar, é feita uma revisão conceitual sobre as diferentes definições e características atribuídas ao estudante e seus desafios.

PALAVRAS CHAVE. Aluno, perfil, desafio e desafio

\section{INTRODUCCIÓN}

A partir de la Declaración de Bolonia (1999), se habla de una educación de calidad, centrada en la formación integral del estudiante. El mundo actual no solo exige profesionales con conocimientos disciplinares, sino que aparte de ello desarrolle habilidades que le permitan enfrentarse a los retos que el mundo presente y venidero depara.

El proyecto Tuning, señala que es de gran importancia que las instituciones educativas centren su esfuerzo en la formación del estudiante, por lo tanto, se debe hacer énfasis en el desarrollo de competencias genéricas, específicas y en los resultados del aprendizaje; es decir, los conocimientos, habilidades y capacidades en el saber, saber hacer y el ser que se espera que el alumno domine y demuestre una vez culmine un proceso de aprendizaje a corto, mediano o largo plazo; ahora bien, la unión de los factores competencias y resultado der aprendizaje, generan flexibilidad y autonomía en la construcción de los currículos (Pérez \& Montes , 2007).

Lo dicho anteriormente, demanda cambiar y replantear aspectos metodológicos, curriculares, didácticos y de roles del docente y el estudiante; por un lado, el docente se debe convertir en guía de aprendizaje y abandonar su protagonismo, para dar mayor importancia al estudiante como constructor de su aprendizaje, lo cual lleva a que éste se convierta en el centro de la clase y no viceversa; esto implica que el alumno trabaje de manera más activa, autónoma, libre, responsable y sea exigente con él mismo (Nolla, Pallés, \& Gual, 2002); ahora bien, estos factores llevan a que el estudiante deba tomar decisiones, argumente y razone; además, sea capaz de reflexionar frente a su propio aprendizaje, se autoevalúe y autocorrija; de ser así, se estaría hablando de verdadera libertad y protagonismo, de empatía, confianza, ultraísmo, autocontrol y responsabilidad estudiantil.

\subsection{Concepto de estudiante}

De acuerdo a la Real Academia de la Lengua Española, estudiante es el participo presente del verbo estudiar; viene del verbo en latín studeo y del griego speudo, el cual significaba 
dedicarse con atención a algo. A partir del siglo I d.C la palabra estudiante se aplicó a las letras y su significado fue el de dedicarse a las letras (litteris studere), es decir estudiar.

El termino estudiante, es un sustantivo de género masculino o femenino que se refiere, en el ámbito académico, al aprendiz que se dedica a estudiar y producir; ahora bien, estudiar, de acuerdo a (Bourdieu \& Passeron , 1967) no es solo producir, sino producirse como capaz de producir, quiere decir ello que estudiar conlleva a serios compromisos de autorregulación, autoeva luación, reflexión y responsabilidad; de hecho, la primera labor de los estudiantes universitarios es educarse en su oficio de "estudiante", en su saber - hacer, ya que tal y como lo expresa (Coulon, 2005) los jóvenes que como alumnos ingresan a la universidad y no logran incorporarse en el mundo de un estudiante, tienden a fracasar.

La palabra alumno se deriva del latín "alumnus" refiriéndose al niño de pecho, que debe ser alimentado; procede del verbo "alere" que significa nutrirse del saber; en ese sentido, el alumno es pasivo, escucha y asimila; por el contrario, el estudiante interroga el saber, lo desenmascara, traga y lo pasa por un proceso de masticación para poder digerirlo de manera más provechosa; desde la anterior mirada, se puede decir que el alumno es aquel que inicia su vida académica (primaria y secundaria); es posible que aún no haya desarrollado a cabalidad el pensamiento crítico, y que, por lo tanto, su formación profesional aun no sea clara o no se tenga definida; es decir, no se lleva en una dirección concreta.

El estudiante, por el contrario, escoge la carrera profesional a partir de su vocación o su madurez educativa, quiere decir esto, que se encuentra en una etapa adulta en la cual se ha desarrollado el pensamiento crítico y autónomo frente a su proceso de aprendizaje, lo cual le debe permitir ajustarse a los métodos y códigos de enseñanza universitarios.
1.2. Características de un estudiante del siglo XXI. Los estudiantes universitarios deben poseer ciertas características, dentro de ellas (De la Cruz, 2003) menciona:

* El carácter autónomo en la exploración de información, capacidad de reflexión y de aplicación de sus conocimientos en la resolución de problemas, su carácter de cooperativismo, trabajo en grupo y especialmente el sentido de responsabilidad el cual es considerado clave para el éxito académico; este último, se ve reflejado en la medida que el estudiante participa en forma activa en las actividades que el docente proponga, cumpla con las tareas asignadas, prepare el material de clase con anterioridad, pregunte, indague, analice, saque conclusiones, resuelva problemas y proponga soluciones.

* Razonamiento crítico, lo cual supone la interpretación y reflexión en torno cada contexto, problema, situación, hecho o realidad que rodea al ser humano, llevando al estudiante a generar y suponer juicios críticos y de valor frente a los mismos.

* Iniciativa, decisión, persistencia, cualidades que generan adaptación ante cualquier obstáculo, contexto o realidad que se presente tanto en el ámbito académico como social; el estudiante asume retos y se adelanta a lo que pueda suceder; en consecuencia, analiza y vislumbra soluciones a los posibles problemas; insiste y no se da por vencido.

* Práctica comunicativa, que incluye la capacidad para codificar y decodificar información; está relacionada con el desarrollo de habilidades lingüísticas, gramaticales y de interacción social, lo cual le permita al estudiante comprender los mensajes recibidos, enviar mensajes coherentes y con intencionalidad claras, desarrollando una comunicación asertiva y eficaz. 
* Capacidad de adaptación por medio de la aplicación de diferentes estrategias para adecuarse a los distintos ambientes, metodologías o didácticas que se generan dentro y fuera del aula académica, esta característica lleva a que el estudiante trabaje individualmente o en equipo, participe en las actividades propuestas dentro y fuera del aula, proponga, debata, exprese su opinión y escuche las opiniones de los otros con respeto.

* Capacidad para solucionar problemas, conlleva a que el estudiante identifique dificultades y proyecte alternativas de solución a partir de los conocimientos adquiridos no solo en su área disciplinar sino también a través de su experiencia de vida.

* Planificación como medio para obtener buenos resultados, quiere decir, que el estudiante organiza sus actividades y las desarrolla teniendo en cuenta los recursos disponibles, el tiempo o el esfuerzo que amerita cada una de ellas.

* Autoconfianza la cual le permite al estudiante aprender a partir de su propio trabajo y esfuerzo(García, 2006).

A la luz de la mirada de (Levine \& Cureton , 1998), los universitarios de hoy se caracterizan por:

* Ser nativos digitales, son los más conectados tecnológicamente, lo que se considera, lleva a que sean solitarios y aislados, razón por la cual, es posible, se les dificulte integrase socialmente, aunque recurren como medio de socialización a las redes sociales, sin embargo, las usan frecuentemente para publicar fotos, hacer comentarios de las fotos que suben otras personas, que muchas veces, no están dentro de sus amistades pero que si están en la red.

* Están inmersos en situaciones y momentos económicos críticos lo que lleva a que el futuro sea incierto y poco claro laboralmente.
* El mundo en que viven es muy diferente al mundo que han vivido sus padres, se encuentran inmersos en mundos disimiles, como resultado hay diferencias de pensamientos y estilos de vida.

* Son temerosos al fracaso y les gusta se les exalte por sus triunfos.

* Son pragmáticos.

* Una de las razones fundamentales para acceder a la educación superior es alcanzar una formación que les permita adherirse a un trabajo y de esa manera obtener un salario que les permita vivir dignamente.

* Para los estudiantes de hoy, el progreso personal y la capacidad para aprender y enfrentar las distintas problemáticas con otras personas o relaciones interpersonales quedan en segundo plano, pues no están en primera línea de interés, de hecho, el cara a cara muchas veces no es tan placentera como solía pasar en épocas anteriores, son poco conversadores, silenciosos, exponen y argumentar sin escuchar.

* Su vida está documentada a través de las redes sociales, de tal manera, parte de su tiempo está dedicado a la conexión digital lo cual permita mantener actualizadas sus redes; por otro lado, las visitas que los estudiantes realizan a las bibliotecas son para encontrar un espacio de sosiego y o para consultas académicas.

\subsection{Perfil del estudiante del siglo XXI.}

La palabra perfil, tiene varias definiciones, dependiendo del área o disciplina a la cual nos referimos, sin embrago en este caso se tomará la definición dada por la Real Academia de la Lengua Española (RAE, 2021) el cual corresponde a: "conjunto de rasgos peculiares que caracterizan a algo o alguien” (pág. 1177). En el ámbito universitario la palabra perfil, se utiliza para definir al profesional de un programa; para tal caso, su concepto es usado 
de manera flexible para indicar el producto o las características que debe poseer un egresado del programa o der la universidad.

De acuerdo a autores como (Arnaz, 1981), un perfil profesional debe destacar el rol de los conocimientos, por tanto, debe describir las características que requiere el futuro profesional para solucionar los posibles problemas y las necesidades sociales con las que se enfrentará ; en ese sentido, propone: definir claramente las áreas en las que el estudiante debe adquirir conocimiento que le permita tener dominio profesional; establecer las actividades que se deben realizar en el área para que se desarrolle el conocimiento necesario y de esa manera el perfil profesional sea idóneo; desarrollar valores y actitudes que le permitan al profesional desempeñarse profesionalmente. Para el caso de (Diaz, Lule, Pacheco, Rojas , \& Saad, 1999) define el perfil profesional como un grupo de conocimientos, actitudes y habilidades que delimitan el ejercicio profesional.

Otro autora importante para mencionar y que aborda el concepto de perfil profesional es (Pérez G. , 1985); para ella, éste define las habilidades, cualidades y conocimientos que debe poseer un estudiante al finalizar sus estudios, por ello , dice la autora que es imprescindible que los planes de estudio planteen estrategias que conduzcan al desarrollo de dichas habilidades; además recalca la necesidad de tener claro los compromisos que el futuro profesional tiene para con la sociedad, para con el sector social en el que está inmerso, con la región y con el mundo.

Bajo otra perspectiva, los estudiantes del siglo XXI son considerados como protagonistas de su aprendizaje, quiere decir esto, que el aprendizaje es un proceso personal en el cual el docente es solo facilitador u orientador del mismo; por su puesto, el protagonismo del estudiante exige que asuma un rol activo y crítico, debe también entender que el único interesado en su aprendizaje es él mismo y no delegar a segundos o terceros sus obligaciones como estudiante; en otras palabras, tal y como lo plantea (Fernández , 1999) el protagonista del aprendizaje se empodera de su proceso, asume una actitud activa, construye significados, pregunta, desarrolla estrategias de estudio, se hace responsable de su aprendizaje; en otros términos, el protagonista de su aprendizaje muestra madurez, autonomía, disciplina y actitud positiva frente a los retos que el sistema educativo, el medio y la sociedad le otorga.

El protagonista de su aprendizaje debe saber que no solo basta con acumular conocimientos en áreas disciplinares, sino que debe reconocer sus capacidades, habilidades y aprovecharlas al máximo, no es el docente que debe descubrirlas, por el contrario, el estudiante debe mostrarlas, por ello no basta con repetir lo que se aprende o se enseña, se debe más bien buscar, confrontar, seleccionar, indagar, criticar, debatir y sacar conclusiones.

El estudiante protagónico de su aprendizaje, aprende a la luz de sus propios logros, es autónomo, tanto así, que se hace responsable de su proceso académico, sin culpar a externos por sus resultados negativos (docentes, currículo, aulas, metodologías y demás factores académicos); asume responsabilidad y se autoevalúa para con ello emprender acciones que le permitan mejorar.

Deacuerdo a (Kamii \& López, 2014), la autonomía es la capacidad de pensar críticamente por uno mismo, en el ámbito moral y docto; dicha autonomía, se adquiere desde los primeros años de vida, es decir, en el momento que se tenga control biológico de las necesidades personales, y, posteriormente pasar a la etapa de autonomía intelectual; en tal sentido, el desarrollo humano es inadmisible sin la libertad de expresión, de opinión, de pensamiento, de filosofía, toda vez que, cada uno de los seres humanos tienen libre albedrío para decidir, hacer, crear o sentir (UNESCO, 2005), sin embrago, en lo que 
respecta a los estudiantes, ser autónomo no quiere decir que el estudiante haga lo que a él le parece bien, sino más bien que sea capaz de tomar decisiones que le representen un cambio, una vivencia o una experiencia importante para su futuro como profesional y su vida laboral o familiar.

Al respecto (Saenz, Galán , \& Luna, 2003) manifiestan que la asistencia a clases debería dejar de ser obligatoria, pues el interés por asistir al aula académica está sujeta al grado de madurez de los estudiantes y a su capacidad para determinar lo que realmente es importante para su formación profesional, no quiere decir esto, que se le adjudique libertinaje; por el contrario, se trata de dar libertad de pensamiento y desarrollar el pensamiento crítico, a través de la aplicación de estrategias didácticas que despiertan y potencien la capacidad analítica lo cual lleva a que él sea un individuo autónomo, capaz de seleccionar información importante y relevante para su formación o por el contrario desecharla.

En esa medida, (Saenz, Galán , \& Luna, 2003), proponen el desarrollo de capacidades analíticas y que conlleve a que los estudiantes se conviertan en seres autónomos de su aprendizaje: en primer lugar mencionan la importancia de dar libertad a la estudiante de tomar apuntes; el docente aclara conceptos y dudas que se presenten o se generen después de leer documentos sugeridos, se debatan temas relacionados con distintas áreas temáticas. La evaluación debe de dejar ser memorística y más bien debe ser de corte analítico, estudios de caso y demás estrategias evaluativas que no mida, sino que cualifique, permitiendo así que el estudiante se exprese de manera libre, muestren su habilidades y competencias. Se debe tener en cuenta que los docentes tienen responsabilidad y compromiso en la generación de autoconocimiento, autoestima y motivación para que como consecuencia de ello el estudiante asuma una posición autónoma (Zuñiga, 2012).

Para (Gerver, 2009), el estudiante de hoy, debe sentir alegría por aprender, por descubrir, por preguntar y hallar respuestas; por consiguiente, es indispensable que él se sienta motivado desde dentro, desde su ser, desde su interior, pues aquel que espera recompensa o estímulos desde el exterior, estará destinado al fracaso, será relegado.

Finalmente, la innovación debe ser una estrategia para desarrollar las actividades, para ello el estudiante le corresponde ser creativo en el desarrollo de las actividades, mostrar habilidades propositivas y de solución de distintos problemas en el área disciplinar.

\subsection{Retos y desafíos de los estudiantes del siglo XXI.}

El estudiante de este siglo, requiere buscar estrategias que de una u otra manera fortalezcan su aprendizaje, al respecto (Mori, 2013.) indica que el estudiante debe:

* Aprender a aprender, es decir, desarrollar capacidad de interpretar la información recibida, procesarla, preguntarse e indagar de manera amplia sobre el mismo.

* Proyectar y resolver problemas, dejando de lado el facilismo y lo común.

* Debatir con argumentos y bases conceptuales o teóricas sin dejar de lado su percepción.

* Aprender a comprender, no acumulando información, sino que por el contrario la información recibida permita reconocer el entorno y actuar bajo las tendencias y exigencias del mismo.

* Aprender a relacionarse, a realizar trabajo colaborativo, entendiendo este como una forma de interacción, un espacio de debate, de construcción de conocimiento y no la simple 
delegación de actividades sin propósito claro, únicamente con la intención de cumplir con la actividad asignada y solo preocupado por la calificación o la evaluación.

* Generar redes de aprendizaje con estudiantes de otras instituciones.

* Usar las redes sociales como herramienta y como medio de formación académica, a través de la formulación de problemas disciplinares en las cuales otras personas interactúen y den posibles soluciones.

* Desarrollar capacidades comunicativas: escuchar, leer y escribir

* Demostrar conducta ética en los contextos personal, laboral y comunitario.

* Autorregularse, responsabilizarse y disciplinarse, proyectando metas a corto, mediano y largo plazo.

Por otro lado, en esta época en la que la cultura digital se ha ido apoderando de todos los contextos: sociales, familiares, laborales, educativos y en la que la plusvalía del conocimiento y las multicompetencias se acentúan de manera más fuerte; es indispensable que el estudiante comprenda, que no basta con el uso constante de las redes sociales, o algunos recursos o herramientas tecnológicas, sino que, más bien, deben afanarse por buscar formas de comunicación que le permitan insertarse profesionalmente en el mundo laboral , toda vez que: "la humanidad de forma acelerada ha ido alterando las formas de comunicación, de entretener, de trabajar, negociar, gobernar y de socializar, sobre la base de la difusión y uso de las Tic a escala global" (García, Portillo, Romo, \& Benito, 2007, pág. 21).

Indudablemente las Tic, han permitido el aumento de la productividad; las grandes, medianas y pequeñas empresas han incorporado recursos tecnológicos que de una $u$ otra manera han generado un crecimiento inimaginable en la producción y la innovación, tal y como lo dice (Pérez C. , 2002, pág. 31) "la humanidad se encuentra en punto de viraje", de tal manera, el estudiante de este siglo debe preparase para retar y recrear el mundo, es consecuencia que se encare con creatividad e innovación, con argumento y con proposición.

Desde el punto de vista anterior, el estudiante del siglo XXI, se enfrenta a desafíos y retos importantes, es el caso del tener que afrontar cantidad de información que día a día se genera en los distintos escenarios sociales; por ello, necesita desarrollar:

* Adaptabilidad para poder alcanzar las metas y los estándares de calidad que el medio, las empresas y el mundo laboral exige.

* Tolerancia para poder escuchar y ser
escuchado

* Proponer y crear y administrar material multimedia, siendo esta una forma de comunicación que atrae a los estudiantes de hoy.

* Estudiar, conducir, constituir, valorar y crear información usando distintos medios de comunicación.

* Adaptarse a roles distintos para de esa manera ejercer empatía

\section{Reflexión en torno al perfil del estudiante del siglo XXI.}

La educación universitaria, sin lugar a dudas ha cambiado debido al vertiginoso y acelerado ritmo social, comunicativo, económico, laboral, político, tecnológico y demás escenarios en los que se ve representado el hombre. Una serie de factores están relacionados a dichos cambios, al respecto (Rodrígez, 2015) (Fielden , 2001) (Levine \& Cureton, 1998), mencionan los siguientes: 
${ }^{*}$ La explosión del conocimiento

${ }^{*}$ Revolución de las comunicaciones

* Estilos de enseñanza y aprendizaje diversos

* El docente deja de ser protagónico y sede su rol protagónico al estudiante

* Ritmos de vida acelerados, tiempo y espacio asimétrico

* Presiones sociales, familiares, laborales y económicos. que generan stress

*El mercado laboral demanda formación profesional con capacidad de resolver problemas, con etnicidad y responsabilidad

Ahora bien, las tecnologías de la información cada día se apropian más de los escenarios y los contextos laborales, sociales, empresariales y académicos, se vive en un mundo interconectado, en el cual la globalización como proceso homogenizado coexiste en medio de un universo con variedad cultural y en constante conflicto y mutación, lo que lleva a que las exigencias del medio sean cada día mayores; en ese sentido, el mercado laboral se ha vuelto más ahogado, los perfiles profesionales que demandan son disimiles a los que en épocas anteriores se necesitaban. En este momento de divergencia, se requiere de profesionales con competencias y habilidades digitales, con creatividad, con proposición y dispuestos a resolver problemas que la sociedad y el mercado insertan; cubriendo así, las necesidades de una sociedad inteligible, vacilante e incierta, al respecto (Bauman, 2044) denomina a esta sociedad "sociedad liquida".

Respecto a las cualidades del estudiante universitario (Gisbert, 2011), denomina a los estudiantes de "hoy", como "digital learners" o "aprendices del nuevo milenio", refiriéndose a la generación de jóvenes estudiantes adictos, activos y creativos en relación a los ordenadores y a las tecnologías; esta generación, procesa la información de manera muy distinta a las anteriores generaciones, de tal manera, (Pedró , 2006), indica que los estudiantes de hoy, acceden a la información por medios digitales, priorizan de igual manera información visual, imágenes y sonidos (restando valor a textos escritos), realizan tareas simultáneamente y procesan información no lineal, intermitente.

Al estudiante del siglo XXI, le corresponde la tarea de desarrollar competencias que van más allá del uso permanente de las tecnologías y las redes sociales, le atañe también cimentar el conocimiento por medio de fuentes digitales, documentos e información que está dispuesta en internet la cual es conveniente analice, critique, confronte, filtre y refute; así mismo, le concierne valorar las herramientas digitales como medio de construcción del conocimiento y entender que su autoformación y autorregulación es el único medio para alcanzar los propósitos de formación profesional y de esa manera poder estar a la par con lo que el contexto y el mundo globalizante demanda.

\section{CONCLUSIONES}

En primera medida es importante resaltar que cuando se habla de estudiante, se refiriere a jóvenes que han ingresado a la educación superior y que han dejado de alumnos para convertirse en personas autónomas y libres de seleccionar la disciplina en la cual quieren profesionalizarse para posteriormente insertarse en el mundo laboral. Así mismo, el estudiante se caracteriza por su capacidad de desarrollarse como ser independiente, activo, participativo, fomentando sus valores éticos y sociales; formándose como ser autónomo libre de elegir, de auto aprender, de exigirse a sí mismo, lo que conlleva a la toma de decisiones en cuanto a tipos de estudio, espacios, fuentes de información, herramientas y demás recursos que seleccione para mejorar su formación, 
convirtiéndose de esta manera en protagonista de su aprendizaje.

Así mismo, el estudiante del siglo XXI, se caracteriza por ser auto reflexivo, lo cual to conlleva a razonar y discrepar de la información que le dan, analiza y argumenta, para que de esa manera su formación se torne en un proceso humanizante y menos mecánica y repetitiva, en otras palabras, el estudiante se convierta en un ser pensante, actuante y creador de su propio aprendizaje, el discernimiento permite de la misma manera interpretar y futurizar el alcance de sus acciones y de su formación.

El estudiante, debe dejar de ser antagonista para ser protagonista de su aprendizaje, reconstruyendo críticamente y participando en la construcción del conocimiento; además se debe preparar para afrontar los retos que la sociedad, el mundo, la economía, la política, pero sobre todo el mundo laboral exige en estos tiempos de aceleramiento tecnológico, de innovación y de crecimiento en la productividad.

\section{REFERENCIAS BIBLIOGRÁFICAS}

Arnaz, J. (1981). Guia para la elaboración de un perfil de egresado. Revista de Educación Superior, 1-7.

Bauman, Z. (2044). Modernidad líquida. Buenos Aires: Fondo de Cultura Económica .

Bourdieu, P., \& Passeron , J. (1967). Los estudiantes y la cultura. Barcelona: Labor.

Coulon, A. (2005). Le métier d'étudiant. L'entrée dans la vie universitaire. Paris: Deuxiéme.
De la Cruz, A. (2003). El procesos de convergencia europeo: ocasión de modernizar la universidad española si se produce un cambio de mentalidad en gestores, profesores y estudantes. Aula Abierta, 191-216.

Diaz, F., Lule, M., Pacheco , D., Rojas , S., \& Saad, E. (1999). Metodología de diseño curricular para educación superior. México: Trillas.

Fernández , A. (1999). Cómo aprender a estudiar con eficiencia y eficacia: nuevo perfil del estudiante del sigloXXI. San Salvador : Editores UCA.

Fielden , J. (2001). Higher Education Staff Development: Continuing Mission. Thematic Debate of the Follow-up to the World Conference on Higher Education. UNESCO.

García, A. (2006). Diseño del aprendizaje basado en competencias. Universidad de Girona.

García, F., Portillo , J., Romo, J., \& Benito, M. (2007). Nativos digitales y modelos de aprendizaje. Post-Proceedings del IV Simposio Pluridisciplinar sobre Diseño, Evaluación y Desarrollo de Contenidos Educativos Reutilizables, (págs. 19-21). Bilbao, Spain.

García, M. (2006). Las competencias de los alumnos universitarios. Revista Interuniversitaria de Formación del Profesorado, 253-269.

Gerver, R. (2009). Craer hoy la escuela del mañana: la educación y el futuro de nuenstros hijos. España: Ediciones S.M. 
Gisbert, M. (2011). Digital Leaners: la competencia digital de los estudiantes universitarios. La Cuestión Universitaria, Vol. 7, 48-51.

Kamii, C., \& López, P. (2014). La autonomia como objetivo de educación: implicaciones de la teoria de Piaget. Infancia y Aprendizaje. Vol. 5. conusltado https://www.researchgate.net/ publication/271943634_La_autonomia_ como_objetivo_de_la_educacion_ Implicaciones_de_la_teoria_de_Piaget, 3-32.

Levine , A., \& Cureton, J. (1998). GAT we know about today's college students. About Campus: Enriching the student learning experience, 4-9.

Levine, A., \& Cureton , J. (1998). we know about today's college students. About Campus: Enriching the student learning experience, (págs. 4-9).

Mori, G. (2013.). El perfil del estuduiante del siglo $X X I$. obtenido de https://www.academia. edu/35478863/el_estudiante_ universitario_del_siglo_XXI

Nolla, M., Pallés, J., \& Gual, A. (2002). Desarrollo de las competencias profesionales. Educación , 76-81.

Pedró , F. (2006). Aprender en el nuevo milenio: Un desafío a nuestra visión de las tecnologías y la enseñanza. OECDCERI.

Pérez, C. (2002). Technological revolutions and financial capital: the dynamics of bubbles and golden. México: Siglo XXI.

Pérez, C., \& Montes , M. (2007). El nuevo rol del profesor y del alumno en el Espacio Europeo de Educación Superior. Archivos de la Sociedad Española de
Oftalmología, vol.82 no.5 , 1-5.

Pérez, G. (1985). El perfil académico profesional Revista Perfiles Educativos .

RAE, D. (15 de marzo de 2021). REA. Obtenido de https://dle.rae.es/perfil

Rodrígez, S. (2015). Los estudiantes universitarios de hoy: una visión multinivel. REDU - Revista de Docencia Universitaria Vol 13, 91-124.

Saenz, L., Galán , W., \& Luna, O. (2003). Reflexiones sobre Educación Superior en México. México : Centro de Estudios Sobre la Universidad, Centro de Innovación y Competitividad de la Universidad Autónoma de León .

UNESCO. (consultado el 11 de marzo de 2021 de 2005). Unesco. Obtenido de https://unesdoc.unesco.org/ark:/48223/ pf0000141908_spa

Zuñiga, M. (2012). Los estudiantes universitarios del siglo XXI en México: de la pasividad a la autonomia y pensamiento critico - Revista Teoria de la educación: Educaión Cultura en la Sociedad de la UInformación. , 424-440. 